\title{
Über Atom- und Molvolume beim absoluten Mullpunkte.
}

\section{Von Richard Lorenz und W. Herz.}

Die Beziehung des Molvolums kristallisierter Körper zu den Volumen der Komponenten ist in neuester Zeit durch die BoRNschen Betrachtungen der energetischen Verhältnisse der Raumgitter von wesentlicher Bedeutung geworden. Schon die Begründer der Lehre des Molvolums, H. KOPP und ScHROEDER, konnten bei festen Stoffen auf gewisse additive Zusammenhänge mit Bestimmtheit hinweisen; aber ebenso sicher ergab sich, dab die Atomrolume nicht als unveränderliche Größen in die Verbindungen eingehen, wenn man erstere im Sinne ihrer gewöhnlichen Definition, nämlich als Gesamtrolnm ${ }^{1}$ ) im freien Zustande anffaBt, wie dies beispielsweise in der Atomvolumkurve geschieht. Diese Erscheinung ist ron I. TraUbe ${ }^{2}$ ) und Rrchards ${ }^{3}$ ) (von diesem in Verbindung mit seiner Theorie der kompressiblen Atome) mehrfach behandelt worden. ${ }^{4}$ ) Die neuesten Betrachtungen über die Volume kristallisierter Körper beziehen sich hauptsächlich auf die Alkalihaloide, weil bei diesen die Berechnungen der Raumgitter am leichtesten zugänglich sind. In erster Reihe wurden dabei die Molvolume der kristallisierten Salze mit den durch Berechnung feststellbaren Volumen der Bestandteile in gebandener Form verglichen, ${ }^{5}$ ) und rom rein chemischen Standpunkte ist dabei eine besonders einfache Regel herrorzuheben, die vor kurzem W. Brutz ${ }^{6}$ ) angegeben hat. Im Gegensatz dazu interessierte uns hier die Frage, wie sich die Molvolume kristallisierter Verbindungen zu den Atomvolumen der freien in ihnen enthaltenen Elemente verhalten. Um diesen Vergleich aber

1) „Raumbeanspruchung" nach W. Brws, Zeitschr. anorg. u. allg. Chem. 115 (1921) 241.

g) Zeitschr. anorg. Chem. 40 (1904), 372.

3) Zeitschr. phys. Ohem. 40 (1902), 169, 597 : 42 (1903), 129; 49 (1904) 15.

4) Vgl. auch Basqa, Phil. Mag. [6] 40 (1920), 169; Rankins, ebenda 516.

5) Bonw, Zeitsehr. f. Physik 1 (1920), 45, 221; HrydwriLLER, ebenda 393; Lorenz, ebenda 2 (1920), 175; Fajams und Grmu, ebenda 299; Fajaks und Hemzrat, ebenda 309.

๑) Zeitschr. anorg. u. allg. Chem. 115. (1921), 241. 
korrekt durchzuführen, ist es notwendig, daB die Volume nicht bei zufälligen und wechselnden Temperaturen betrachtet werden, sondern daB eine charakteristische Temperatur festgelegt wird. Es ist das ein Punkt, der bei allen bisherigen Untersuchungen über Atom- und Molvolume keine gentugende Berücksichtigung gefunden hat (wenn wir von den RegelmäBigkeiten der Molvolume von Flüssigkeiten absehen, die hier nicht direkt in Betracht kommen). Schon vor einiger Zeit hatte der eine von uns (HEnz) in einer Abhandlung über die Nullpunktsdichten einiger Elemente ${ }^{1}$ ) darauf hingewiesen, daß die seit Lothar Mexer berühmte Kurve der Atomvolume und Atomgewichte erst dann als wirklich prinzipiell definiert angesehen werden kann, wenn es sich um charakteristische Temperaturen handelt, bei denen die zur Berechnung der Atomvolume benutzten Dichten bestimmt worden sind. Es ist vielleicht nicht ganz ohne Interesse, an einem extremen Beispiele zu zeigen, wie groB die Differenzen in den Atomrolumen bei verschiedenen Temperaturen sein können. Beim Argon ist die Dichte beim Siedepunkte zu 1,212 und die kritische Dichte zu 0,509 bestimmt; die Dichte beim absoluten Nullpunkte ist in der zitierten Abhandlung von HERz zu 1,709 berechnet worden, und daraus folgen:

die Atomvolume beim absoluten Nullpunkte . . 23,34

beim Siedepunkte . . . . . 32,90

bei der kritischen Temperatur 78,35

Die Werte schwanken um mehr als das Dreifache, und man kann nicht sagen, daß eine dieser Zahlen die Eigenschaften des Argons am besten wiedergibt. Bei völliger Freiheit der Auswahl würden bei Verwendung dieser Zahlen Ungenauigkeiten entstehen. ${ }^{2}$ ) Wenn nun auch bei sehr vielen Elementen, insbesondere den bei gewöhnlicher Temperatur festen, derartige Schwankungen des Atomvolums vielleicht praktisch nicht berücksichtigt zu werden brauchen, so muB doch grundsätzlich zugegeben werden, daB die Einführung vergleichbarer Verhältnisse bei derartigen Berechnungen jedenfalls nützlich sein könnten.

Als besonders zweckmäßig könnten die Verbältnisse beim absoluten Nullpunkt hierzu erscheinen. Alle Stoffe würden dann bei derselben Temperatur betrachtet werden und unter vergleichbaren

1) Zeitschr, anorg. u. allg. Chem. 105 (1919), 171.

2) Eine weitere Schwierigkeit ist die Verschiedenheit der Dichte bei den verschiedeneu allotropen Modifikationen der Elemente. 
Zustandsbedingungen stehen. Die wahren Volume sind nun aber beim absoluten Nullpunkt unbekannt. Es liegt jedoch nahe an Stelle des wirklichen Nullpunktes den absoluten Nullpunkt im Sinne der Regeln der übereinstimmenden Zustände einzuführen, die uns zwar nicht die wirklichen Verhältnisse, wohl aber eine Annäherung an diese im Sinne von Mittelwerten aus einer sehr großen Zahl ron Stoffen ergeben. ${ }^{1}$ )

Die Berechnung der Nullpunktsdichten in dem vorher erwähnten Sinne bietet bei Herbeischaffung genügenden Materials keine Schwierigkeiten, wenn man die Beziehungen berücksichtigt, die LoRENz ${ }^{2}$ ) zwischen der Dichte beim ,absoluten Nullpunkt" und den Dichten in anderen Zuständen zusammengefaßt hat.

In der zitierten Abhandlung von HERz sind die Atomvolume und Dichten einiger Elemente beim absoluten Nullpunkt angegeben worden, und vor kurzem haben wir $^{3}$ ) die Dichten einiger Salze beim absoluten Nullpunkt in eben demselben Sinne berechnet, woraus ohne Weiteres die Molrolume bei dieser Temperatur folgen. Man kann nun diese Molvolume $\left(M V_{0}\right)$ mit der Summe der Nullpunktsatomrolume der die Salze zusammensetzenden Atome $\left(\Sigma A P_{0}\right)$ vergleichen, und die Tabelle zeigt das Ergebnis:

\begin{tabular}{|c|c|c|c|c|c|c|}
\hline & & & & $\boldsymbol{M} V_{0}$ & $\Sigma A V_{0}$ & "Kontraktion" \\
\hline $\begin{array}{l}\text { Natriumfluorid } \\
\text { Natriumchlorid } \\
\text { Natriumjodid . }\end{array}$ & $::$ & $:$ & i. & $\begin{array}{l}17,76 \\
31,12 \\
45,24\end{array}$ & $\begin{array}{l}33,66 \\
37,41 \\
45,53\end{array}$ & $\begin{aligned} 15,90 & =47,3 \% \\
6,29 & =16.8 \% \\
0,29 & =0,6 \%\end{aligned}$ \\
\hline $\begin{array}{l}\text { Kaliumfluorid } \\
\text { Ratiumchlorid } \\
\text { Kaliumjodid . }\end{array}$ & $\begin{array}{ll}. & . \\
. & \cdot \\
. & .\end{array}$ & $:$ & : & $\begin{array}{l}25,16 \\
40,30 \\
55,97\end{array}$ & $\begin{array}{l}51,36 \\
55,11 \\
62,23\end{array}$ & $\begin{aligned} 26,20 & =51,1 \% \\
14,81 & =26,8 \% \\
7,26 & =11,5 \%\end{aligned}$ \\
\hline $\begin{array}{l}\text { Cäsinmfluorid } \\
\text { Ctsiumehlorid } \\
\text { Cxsiumjodid . }\end{array}$ & 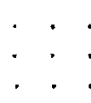 & $:$ & . & $\begin{array}{l}34,48 \\
49,81 \\
67,08\end{array}$ & $\begin{array}{l}86,57 \\
90,32 \\
98,44\end{array}$ & $\begin{array}{l}52,09=60,0 \% \\
40,51=44,9 \% \\
31,36=31,9 \%\end{array}$ \\
\hline $\begin{array}{l}\text { Natriumnitrat . } \\
\text { Kaliumnitrat : } \\
\text { Cassiumnitrat : }\end{array}$ & $\begin{array}{l}\cdot \\
\dot{\cdot} \\
\cdot\end{array}$ & $:$ & & $\begin{array}{l}36,78 \\
44,72 \\
57,30\end{array}$ & $\begin{array}{r}64,16 \\
81,36 \\
117,07\end{array}$ & $\begin{array}{l}27,38=42,5 \% \\
36,64=45,1 \% \\
59,77=50,7 \%\end{array}$ \\
\hline $\begin{array}{l}\text { Natriumsulfat . } \\
\text { Kaliumsulfat } \\
\text { Cäsiumsulfat : }\end{array}$ & $: \cdot$ & $\cdot:$ & & $\begin{array}{l}56,71 \\
76,16 \\
97,99\end{array}$ & $\begin{array}{r}97,91 \\
133,31 \\
203,73\end{array}$ & $\begin{aligned} 41,20 & =42,1 \% \\
57,15 & =42,7 \% \\
105,74 & =51,9 \%\end{aligned}$ \\
\hline
\end{tabular}

1) Vgl. hierüber die Ausführungen über den ,idealen physikochemischen Stoff" von R. LoBENz, Zeitsehr. anorg. u, allg. Chem. 94 (1916), 240; 103 (1918), 243.

2) Zeitschr, anorg. u. allg. Chem. 94 (1916) 240.

s) Zeitschr. anorg. u. allg. Chem. 117 (1921), 103. 
In allen Fällen ist das Molvolum kleiner als die Summe der Komponenten. Wir haben das Verkleinerungsverhältnis in Prozenten in der letzten Spalte der Einfachheit halber als "Kontraktion" bezeichnet, wie dies vielfach in der Literatur üblich ist. Es muB. jedoch ausdrücklich darauf aufmerksam gemacht werden, dab die Auffassung dieser Verkleinerungen im Sinne einer Kontraktion oder wenigstens ausschlieBlich einer solchen nicht berechtigt sein dürfte und auch in diesem Sinne hier nicht untersucht werden soll. Vergleichen wir analoge Natrium-, Kalium- und Cäsiumverbindungen, so ist immer die „Kontraktion“ beim Cäsium am gröBten und beim Natrium am kleinsten; das Kalium steht in der Mitte. Vergleichen wir die Halogensalze eines Alkalimetalls, so ist die Kontraktion beim Fluor am gröBten und beim Jod am kleinsten. Da das Cäsium das stärkste Alkalimetall und das Fluor das stärkste Halogen ist, während das Natrium das schwächste der hier benutzten Alkalimetalle und das Jod das schwächste Halogen ist, so kann man sagen, daB die Verkleinerung der Atomvolume zum Molvolum der Verbindung um so größer ist, je aktiver die reagierenden Elemente sind.

Wenn auch diese Ergebnisse im einzelnen nicht neu sein mögen ${ }^{1}$ ), so ist doch hervorzuheben, daß hier zum ersten Male alle Volumangaben bei dem gleichen übereinstimmenden Zustande festgestellt worden sind und der Vergleich der Mol- und Atomvolume damit einwandfrei durchgeführt ist.

1) Vgl. auch Ephraim und Mrohed, Helv. chim. Acta 2 (1919), 266.

Frankfurt a. M., Institut für physikalische Chemie der Universität und des physikalischen Vereins.

Breslau, Universität, Physikalisch-chemische Abteilung, 29. März 1921.

Bei der Redaktion eingegangen am 31. März 1921. 